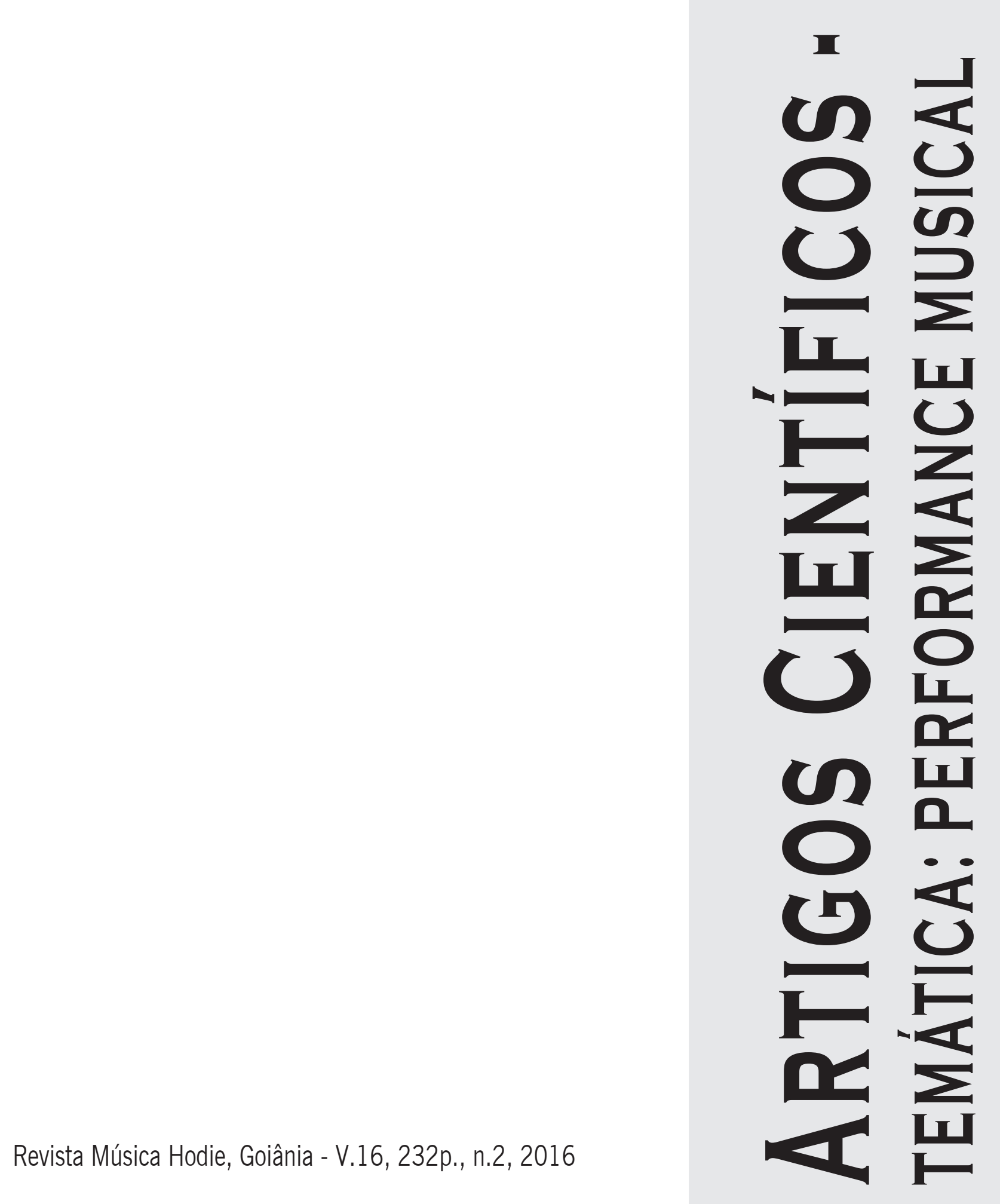




\title{
Como será que eu afino? A relação entre sistemas de afinação e parâmetros de afinação na performance musical
}

\author{
Ricardo Dourado Freire (Departamento de Música, Universidade de Brasília, Brasília, DF, Brasil) \\ freireri@unb.br
}

\begin{abstract}
Resumo: A afinação é uma questão central para a prática musical de vários instrumentistas e cantores. As práticas de afinação ocorrem a partir da definição de parâmetros de afinação que orientam todo o processo. Este artigo propõe um parâmetro de afinação presente na estrutura de afinadores eletrônicos, que estão organizados de acordo com uma frequência de referência de afinação em Hertz $(F R H z)$, usualmente a nota Lá $=440 \mathrm{~Hz}$. Cada parâmetro de afinação oferece recursos para que seja possível identificar a altura de uma nota dentro de uma escala musical. Um sistema de afinação estabelece a organização das frequências de uma escala de acordo com princípios determinados por cada proposta. Assim, o objetivo deste texto é relacionar parâmetros de afinação com sistemas de afinação, e mostrar como um sistema de afinação pode ser realizado na prática. Os resultados demonstram que existem variações específicas em todos os graus da escala quando estão sendo utilizados os sistemas Pitagórico, Justo e Igual. A partir da análise das tabelas, torna-se possível estabelecer referenciais para que instrumentistas possam ajustar e aprimorar sua prática musical na performance dos diferentes sistemas de afinação.
\end{abstract}

Palavras-chave: Parâmetros de afinação; Sistemas de afinação; Acústica e performance musical.

How should I develop good intonation? The relationship between temperament systems and tuning procedures in musical performance

Abstract: Intonation is a central aspect of music performance for instrumentalists and singers, as tuning practices were established based on acoustic and mathematical parameters. This article proposes the use of a new parameter the $\mathrm{FRHz}$ (in Portuguese), based on variations on the standard tuning parameter used in electronic devices. Each tuning parameter establishes the resources to identify pitch frequencies and how the pitches should be organized in a scale. This article aims to identify how tuning parameters relate to each other, and how a musician could apply it to music performance. Results show that each scale degree presents a slight difference when they should be tuned according to Pythagorean, Just or Equal Temperaments. Based on the charts, it is possible to propose references that could guide musicians to adapt the intonation to different temperaments.

Keywords: Intonation; Tuning and temperament; Acoustics; Music performance.

¿Cómo afino yo? La relación entre sistemas temperados y parámetros de afinación en la ejecución musical

Resumen: La afinación es un aspecto central en la ejecución musical de varios instrumentistas y de cantores. Las prácticas de afinación se fundamentan en procedimientos y parámetros acústicos y matemáticos establecidos históricamente por los sistemas Pitagórico, Justo e Igual (o de temperamento igual). Cada sistema de afinación establece parámetros para identificar la frecuencia de la altura de una nota y su organización en la escala. El presente artículo propone un nuevo parámetro usando la variación de la frecuencia de referencia en Hertz $(F R H z)$ de los afinadores electrónicos. El estudio conducido tiene por objetivo identificar como los diferentes parámetros de afinación se interrelacionan y como los músicos pueden aplicar esos parámetros en la ejecución musical. Los resultados muestran que cada grado en una escala presenta variaciones que requieren padrones afinaciones diferentes de acuerdo con los modelos de afinación. De esa forma, fueron elaboradas tablas de frecuencia que clasifican las referencias para orientar las prácticas musicales, posibilitando adaptar la afinación a los diferentes temperamentos.

Palabras clave: Parámetros de afinación; Sistemas de afinación; Acústica; Ejecución musical.

As quintas das cordas soltas nos instrumentos de cordas são quintas justas, mas torna-se impossível que todas as outras quintas sejam justas quando se toca em diferentes tonalidades. Desta maneira, as escalas nas diferentes tonalidades terão diferenças de afinação, e isto torna-se importante e adquire necessariamente uma influência muito grande no caráter das melodias. Sendo que estas qualidades de sonoridade de notas diferentes são muito mais profundas nos instrumentos de sopro. Se esta visão estiver correta, as características das tonalidades devem ser muito diferentes nos distintos instrumentos, e eu acredito que este seja o caso, mas esta matéria deverá ser decidida por um músico com ouvidos delicados, que direciona sua atenção para os questionamentos colocados aqui. (HELMHOLTZ, 1895, p. 311) 
A afinação é uma questão central para a prática musical de todo instrumentista ou cantor. No entanto, a avaliação da afinação musical apresenta um paradoxo: as alturas das notas são estabelecidas a partir de definições exatas e objetivas, como frequências, razões ou cents, mas todo o processo sonoro é avaliado subjetivamente pela percepção humana. O filósofo Aristoxenus já apresentava, no séc. IV A.C., o dilema entre aspectos objetivos e subjetivos ao mencionar que "será que as cogitações de teóricos são tão importantes quanto as observações dos próprios músicos? Ou será que o julgamento dos intervalos realizado pelo ouvido é mais importante que as razões matemáticas?.” (BARBOUR, 2004) No caso da performance musical, cabe ao músico estimular o seu próprio refinamento perceptivo para desenvolver o ouvido "delicado" proposto por Helmhontz (1895), de acordo com o julgamento realizado pelas suas observações práticas. Desta maneira, o processo de afinação torna-se uma habilidade de fazer distinções sutis na organização das frequências das notas dentro de um contexto que pode conter um ou vários instrumentos musicais. ${ }^{1}$ A subjetividade do músico manifesta-se nos pequenos ajustes nas frequências das notas no seu instrumento, que precisam ser modificadas para entrar em concordância com as frequências das notas de outros instrumentos. A correlação entre conhecimento objetivo e subjetividade perceptiva oferece os requisitos e os desafios para um músico demonstrar sua capacidade de afinação em diversos contextos.

A escala musical é utilizada para representar uma tonalidade, uma sequência fixa de intervalos e também para estruturar um sistema de afinação. De acordo com Thompson (2013):

o conceito de escala (musical) pode ser definido a partir de uma perspectiva física, matemática ou psicológica. Na perspectiva física, ela se refere a um conjunto de alturas que podem ser produzidos em um instrumento de acordo com um sistema de afinação. Na perspectiva matemática, pode-se utilizar a descrição de um grupo teórico de notas (pitch sets), como forma de acessar os recursos disponíveis em um sistema de afinação, como por exemplo o sistema igual. Na perspectiva psicológica, uma escala se refere a um conjunto de representações mentais, de uma sequência regular de alturas, que são ativadas quando se ouve música. (p. 127)

As diferentes perspectivas da escala apresentada por Thompson servem de referência para a construção de um paradigma sonoro que irá permitir o desenvolvimento dos elementos estéticos na interpretação musical. No entanto, o músico prático se defronta com algumas questões: Quais são os parâmetros que eu devo seguir para conseguir afinar meu instrumento? Qual sistema de afinação está sendo usado e como eu devo me adaptar a este contexto musical? Afinal, como será que eu devo afinar quando estou tocando?

As práticas de afinação ocorrem a partir da definição de parâmetros de afinação que orientam todo o processo. Um parâmetro de afinação estabelece uma referência para a organização das frequências musicais, enquanto que um sistema de afinação organiza uma sequência fixa de frequências, a escala musical. Um sistema de afinação pode ser descrito por vários parâmetros de afinação, e este artigo tem por objetivo identificar as relações existentes entre as formas de identificação de frequências utilizando razões matemáticas conforme identificadas por Pitágoras e Ptolomeu, o sistema de Cents criado por Alexander Ellis, e a proposta de um parâmetro de comparação vinculado a uma Frequência de Referência de Afinação em Hertz $(F R H z){ }^{2}$ Assim, o propósito deste texto é relacionar parâmetros de afinação com sistemas de afinação e demonstrar como as diferenças entre sistemas de afinação podem ser aplicadas na prática.

A proposta metodológica consiste na análise das descrições das representações de afinação dos sistemas Pitagórico, Justo e Igual. A partir das tabulações das diferentes descrições, é possível determinar quais ajustes de afinação devem ser realizados para a performance de acordo com os sistemas apresentados. 


\section{Parâmetros de afinação e sistemas de afinação}

A estrutura de um sistema de afinação depende da descrição exata das frequências de cada uma das notas de uma escala e de quais são os critérios e os parâmetros para a construção desta escala. A organização de cada um dos sistemas apresentados foi realizada a partir da aceitação de parâmetros específicos de afinação. O Sistema Pitagórico e o Sistema Justo são descritos a partir de razões matemáticas, enquanto o Sistema Igual é descrito com precisão pela divisão da escala em 1200 partes iguais (cents).

O desenvolvimento de um sistema de afinação é consequência dos procedimentos para a estruturação do conjunto de notas dentro de uma escala musical. Durante a história da música, foram desenvolvidos vários procedimentos para estabelecer os critérios de organização das escalas musicais, sendo que a definição de frequências necessita ser realizada a partir de parâmetros fixos. Pitágoras realizou seus cálculos a partir do comprimento de cada uma das cordas do Monocórdio. A análise do comprimento de cada corda demonstrou ser um procedimento prático e exato. Além de permitir a determinação de valores específicos para o comprimento de cada corda, possibilitou o estudo das proporções entre os comprimentos das cordas. Assim, foram determinadas indicações numéricas representando a proporção entre identificação de diversos intervalos musicais, as razões.

A sistematização no uso das proporções matemáticas criou o primeiro parâmetro para determinação dos valores das frequências musicais, as razões (ratios, em inglês). Cada razão determina um intervalo musical e estabelece a proporção nos valores de uma frequência em relação a outra frequência. A partir de uma dada frequência torna-se possível identificar a frequência de outra nota do intervalo multiplicando o valor apresentado pela razão específica de cada intervalo. Por exemplo, para encontrar a oitava justa ascendente da nota Lá3 $=442$, faz-se necessário multiplicar esse valor por 2:1, com resultado Lá $4=884$. Para encontrar o valor da oitava descendente, multiplica-se o mesmo valor por 1:2, chegando à frequência Lá2 $=221$. Outros exemplos: a razão 3:2 indica um intervalo de quinta justa ascendente; a razão 2:3 indica quinta justa descendente; a proporção 4:3 indica uma quarta justa ascendente; a proporção 3:4 indica a quarta justa descendente. A segunda maior ascendente é representada pela razão 9:8; enquanto o mesmo intervalo descendente é representado por 8:9. A proporção entre intervalos e razões pode ser verificada na tabela a seguir (Tabela 1):

Tabela 1: Relação das razões numéricas dos intervalos ascendentes e descendentes.

\begin{tabular}{|l|c|c|c|c|c|c|c|c|c|c|c|c|}
\hline \multicolumn{1}{|c|}{ Intervalo } & $\begin{array}{c}8^{a} \\
\text { justa }\end{array}$ & $\begin{array}{c}5^{a} \\
\text { justa }\end{array}$ & $\begin{array}{c}4^{\mathrm{a}} \\
\text { justa }\end{array}$ & $\begin{array}{c}3^{\mathrm{a}} \\
\text { maior }\end{array}$ & $\begin{array}{c}3^{\mathrm{a}} \\
\text { menor }\end{array}$ & $\begin{array}{c}6^{\mathrm{a}} \\
\text { maior }\end{array}$ & $\begin{array}{c}6^{\mathrm{a}} \\
\text { menor }\end{array}$ & $\begin{array}{c}2^{\mathrm{a}} \\
\text { maior }\end{array}$ & $\begin{array}{c}2^{\mathrm{a}} \\
\text { menor }\end{array}$ & $\begin{array}{c}7^{\mathrm{a}} \\
\text { maior }\end{array}$ & $\begin{array}{c}7^{\mathrm{a}} \\
\text { menor }\end{array}$ & $\begin{array}{c}4^{\mathrm{a}} \\
\text { aum }\end{array}$ \\
\hline Razão ascendente & $2: 1$ & $3: 2$ & $4: 3$ & $5: 4$ & $6: 5$ & $5: 3$ & $8: 5$ & $9: 8$ & $25: 24$ & $15: 8$ & $9: 5$ & $45: 32$ \\
\hline Razão descendente & $1: 2$ & $2: 3$ & $3: 4$ & $4: 5$ & $5: 6$ & $3: 5$ & $5: 8$ & $8: 9$ & $24: 25$ & $8: 15$ & $5: 9$ & $32: 45$ \\
\hline
\end{tabular}

\section{Sistema Pitagórico}

Os estudos iniciais sobre as frequências e as proporções entre as frequências musicais estão atribuídos ao filósofo e matemático grego Pitágoras, que viveu no séc. V A.C. A escala Pitagórica é construída a partir da razão 3:2 quando aplicada na afinação de intervalos de $5^{\mathrm{a}}$ Justa ascendente ou 2:3 na afinação de $5^{\mathrm{a}}$ Justas descendentes, e na utilização da razão 4:3 na afinação de intervalos de $4^{\mathrm{a}}$ Justa ascendente ou 3:4 na afinação de $4^{\mathrm{a}}$ Justas descendentes. A partir da nota Lá3, é possível afinar uma sequência ascendente, seguindo as razões indicadas: Lá3-Mi4 (3:2), Mi4-Si3 (3:4), Si3-Fá\#4 (3:2), Fá\#4-Dó\#4 (3:4), Dó\#4-Sol\#4 (3:2), Sol\#4-Ré\#4 (3:4). Tendo como base a nota Lá4, identificam-se as frequências dos intervalos descen- 
dentes, seguindo as razões indicadas: Lá4-Ré4 (2:3), Ré4-Sol4 (4:3), Sol4-Dó4 (2:3), Dó4-Fá4 (4:3), Fá4-Sib3 (2:3). As razões apresentadas para descrever cada intervalo da escala Pitagórica são consequências da sequência de razões utilizadas a partir do ciclo de quintas. (Tabela 2)

Tabela 2: Escala Pitagórica representada por razões e frequências.

\begin{tabular}{|c|c|c|c|c|c|c|c|c|c|c|c|c|c|}
\hline \multicolumn{14}{|c|}{ Escala Pitagórica } \\
\hline & Lá 3 & Sib3 & $\mathrm{Si3}$ & Dó4 & Dó\# 4 & Ré 4 & Ré\# 4 & Mi 4 & Fá4 & Fá\# 4 & Sol 4 & Sol\# 4 & Lá 4 \\
\hline & $1 \mathrm{~J}$ & $2 m$ & $2 M$ & $3 m$ & $3 \mathrm{M}$ & $4 \mathrm{~J}$ & $4 \mathrm{~A}$ & $5 \mathrm{~J}$ & $6 m$ & $6 \mathrm{M}$ & $7 \mathrm{~m}$ & $7 \mathrm{M}$ & $8 \mathrm{~J}$ \\
\hline Razão & 1 & $256: 243$ & $9: 8$ & $32: 27$ & $81: 64$ & $4: 3$ & $729: 512$ & $3: 2$ & 128:81 & $27: 16$ & $16: 9$ & $243: 128$ & 2 \\
\hline Freq. & 442,00 & 465,65 & 497,25 & 523,85 & 559,41 & 589,33 & 629,33 & 663 & 698,47 & 745,88 & 785,78 & 839,11 & 884 \\
\hline
\end{tabular}

\section{Sistema de afinação justa}

O sistema de afinação justa foi inicialmente proposto pelo matemático e astrônomo Claudius Ptolomeu (90-167), quem defendia que "a melhor afinação é aquela na qual o ouvido e a razão matemática estão de acordo” (BARBOUR, 2004). Posteriormente, Gioseffo Zarlino (1517-1590) organizou uma escala de 17 notas, em que existe uma clara diferença entre sustenidos e bemóis que ilustram as diferentes relações de afinação advindas das razões presentes na série harmônica. A proposta do sistema justo busca identificar as razões que possam descrever com maior clareza um intervalo, baseada na coincidência das frequências de harmônicos presentes na série harmônica de duas notas soando simultaneamente (Tabela 3). O intervalo de $5^{\mathrm{a}}$ Justa é representado pela razão 3:2, enquanto a $4^{\mathrm{a}}$ Justa está representada por 4:3, $2^{\text {a }}$ Maior por 9:8, $3^{\text {a }}$ Maior por 5:4, $6^{\text {a }}$ Maior por 5:3 e a $7^{\text {a }}$ Maior por 15:8, para indicar a escala Maior. A escala menor natural é representada pelos intervalos 3:2 para $5^{\mathrm{a}}$ Justa, 4:3 para $4^{\mathrm{a}}$ Justa, 6:5 para $3^{\mathrm{a}}$ menor, 8:5 para $6^{\mathrm{a}}$ menor, 9:5 para $7^{\mathrm{a}}$ menor, sendo que a $2^{\mathrm{a}}$ menor é representada pela razão 16:15 e a $4^{\mathrm{a}}$ aumentada por 45:32.

Tabela 3: Escala Justa representada por razões e frequências.

\begin{tabular}{|c|c|c|c|c|c|c|c|c|c|c|c|c|c|}
\hline \multicolumn{14}{|c|}{ Escala Justa } \\
\hline & Lá 3 & Sib3 & $\mathrm{Si3}$ & Dó4 & Dó\# 4 & Ré 4 & Ré\# 4 & Mi 4 & Fá4 & Fá\# 4 & Sol 4 & Sol\# 4 & Lá 4 \\
\hline & $1 \mathrm{~J}$ & $2 m$ & $2 M$ & $3 m$ & $3 \mathrm{M}$ & $4 \mathrm{~J}$ & $4 \mathrm{~A}$ & $5 \mathrm{~J}$ & $6 m$ & $6 \mathrm{M}$ & $7 \mathrm{~m}$ & $7 \mathrm{M}$ & $8 \mathrm{~J}$ \\
\hline Razão & 1 & $16: 15$ & $9: 8$ & $6: 5$ & $5: 4$ & $4: 3$ & $45: 32$ & $3: 2$ & $8: 5$ & $5: 3$ & $9: 5$ & $15: 8$ & 2 \\
\hline Freq. & 442 & 471,47 & 497,25 & 530,40 & 552,50 & 586,33 & 621,56 & 663 & 707,20 & 736,67 & 795,60 & 828,75 & 884 \\
\hline
\end{tabular}

\section{Sistema igual}

O sistema de afinação igual, chamado em inglês de equal temperament, refere-se ao procedimento de divisão da oitava musical em doze partes exatamente iguais. Este procedimento teve sua primeira proposta no séc. XVI com Giovanni Maria Lanfranco em sua estruturação dos procedimentos para a afinação de monocórdios e órgãos (BARBOUR, 2004). Durante o final do período romântico, a expansão das possibilidades de modulação e a ampliação no uso de vários centros tonais colocaram em questão o uso de sistemas irregulares ou que privilegiassem determinadas tonalidades. No início do séc. XX, o advento da música atonal exigiu um sistema de afinação no qual todos os intervalos fossem rigorosamente iguais e que apresentasse uma proporção equidistante entre cada nota da escala.

O matemático inglês Alexander J. Ellis (1814-1890) realizou uma série de estudos sobre escalas de diversas culturas ocidentais e orientais. Durante suas pesquisas etnográficas, Ellis (1884) precisou estabelecer um sistema de descrição da relação entre as notas das 
escalas de diversos países para poder identificar as mínimas diferenças entre as alturas das notas. A escolha de utilização do fator $\sqrt[12]{2}=2^{\frac{1}{12}}=1.059463$ permitiu a definição da distância padrão entre cada um dos semitons de uma escala de doze notas. Para a identificação de intervalos, pode-se modificar o fator de cálculo para indicar o número de semitons na fração da potência de dois, como, por exemplo, $2^{\frac{2}{12}}, 2^{\frac{3}{12}}, 2^{\frac{4}{12}}, 2^{\frac{5}{12}}, 2^{\frac{6}{12}}, 2^{\frac{7}{12}}, 2^{\frac{8}{12}}, 2^{\frac{9}{12}}, 2^{\frac{10}{12}}$, ou $2^{\frac{11}{12}}$. O sistema de cents de Ellis contribuiu para fundamentar pesquisas tantos na área de matemática aplicada quanto nas áreas de acústica, musicologia e etnomusicologia (STOCK, 2007).

Ellis (1884) descreve seu método da seguinte maneira:

Mas a simples demonstração do número de vibrações, ou o comprimento de cordas vibrantes para a produção de uma escala, não apresenta nenhum sentido musical para um músico. O músico quer saber quantos semitons igualmente temperados, ou partes destes semitons, estão contidos em um intervalo, de maneira que ele possa compreender, em comparação com as notas do piano moderno, que deverá estar afinado de acordo com o temperamento igual. Esta transformação é facilmente demonstrada pela tabela a seguir (Tabela 4), na qual eu uso cent como a centésima parte do semitom temperado de maneira igual, sendo que existem 12 semitons em uma oitava. (p. 368-369)

Tabela 4: Tabela em Cents (com indicação de razões) apresentada por Ellis no artigo Tonometric Observations on some existing Non-harmonic Music Scales (1884).

\begin{tabular}{|c|c|c|c|}
\hline Intervals. & Cents. & Interrals. & Cents. \\
\hline The Skhisma $\ldots \ldots \ldots \ldots \ldots$ & 2 & Jast major Third, $\frac{5}{4}$. & 386 \\
\hline The Comma of Didymus, $\frac{81}{80}$ & $2 \overline{2}$ & Py thagorean major'Third, $\frac{81}{64}$ & 4,08 \\
\hline The Pythagorean Comma .. & 24 & 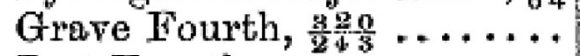 & 476 \\
\hline The Septimal Comma, $\frac{64}{8} \ldots$ & 27 & Just Fourth, 告........... & 4.98 \\
\hline 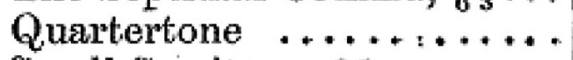 & 50 & Septimal Fifth, $\frac{7}{5} \ldots \ldots$ & 583 \\
\hline Small Semitone, $\frac{25}{2} \ldots \ldots \ldots$ & 70 & Tritone, $\frac{45}{3} \ldots \ldots \ldots$ & 590 \\
\hline Pythagorean Limma, $\frac{25}{2} \frac{5}{3} \ldots$ & 90 & Grave Fifth, $\frac{4}{2}, \ldots \ldots$ & 680 \\
\hline Small Limma, $\frac{13}{12} 5 \ldots \ldots$ & 92 & Just Fifth, $\frac{3}{2} \ldots \ldots \ldots$ & 702 \\
\hline Diatonic Semitone, $\frac{16}{15} \ldots \ldots$ & 112 & Acute Fifth, $\frac{29.3}{160} \ldots$. & 724 \\
\hline Pythagorean Apotome..... & 114 & Just minor Sixtl, $\frac{8}{5}$.. & 814 \\
\hline Great Limma, $\frac{27}{2}, \ldots \ldots \ldots$ & 134 & Just major Sixth, $\frac{5}{3} \ldots \ldots \ldots$ & 884 \\
\hline The Trumpet $\frac{3}{4}$ T'one, $\frac{1}{1} \frac{2}{1} \ldots$ & 151 & Pythagorean major Sixth, $\frac{27}{16}$ & 906 \\
\hline The minor Second, $\frac{100}{9} \ldots \ldots$ & 182 & Natural minor Seventh, $\frac{z}{4} \ldots$ & 969 \\
\hline The major Second, 量...... & 204 & Minor Seventh, $\frac{10}{y}, \ldots \ldots \ldots$ & 996 \\
\hline Septimal minor ' 1 'hird, $\frac{7}{1} \ldots$ & 267 & Just major Seventh, $\frac{15}{8} \ldots$ & 1088 \\
\hline Pythagorean minor Third, $\frac{3}{2} \frac{3}{7}$ & 294 & Pythag. major Serenth, $\frac{24}{12}$ & 1110 \\
\hline Just minor Third, $\frac{6}{5} \ldots \ldots \ldots$ & 316 & 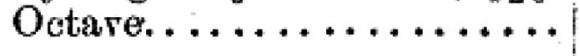 & 1200 \\
\hline
\end{tabular}

Pode-se notar que Ellis criou um sistema destinado ao músico prático, para o qual fosse possível observar e realizar os ajustes de afinação com precisão de um centésimo de semitom. A explicação matemática articulada à demonstração das proporções entre os diversos intervalos da escala permitiu que o sistema de Ellis fosse amplamente adotado e difundido em toda a cultura acadêmica ocidental. No entanto, a visão prática do trabalho de Ellis perdeu o vínculo com a performance musical, pois ficou fortemente vinculada a descrições teóricas. Enfatizo aqui que o objetivo de Ellis foi plenamente desenvolvido em áreas teóricas principalmente na descrição de sistemas de culturas específicas, e cabe aos instrumentistas encontrar maneiras de utilização do sistema de Cents nas práticas de performance atuais. 
A música para instrumentos de afinação fixa e principalmente a afinação do piano exigem um sistema definido e estável para afinação de todas as cordas do instrumento. Além disso, o processo de construção de violões e de instrumentos de cordas com trastes precisa seguir rigorosos procedimentos de colocação na divisão entre as notas para permitir uma afinação proporcional em todas as tonalidades (Tabela 5). O Sistema Igual propõe um parâmetro objetivo que se tornou o padrão internacionalmente aceito na cultura musical ocidental no séc. XX.

Tabela 5: Escala cromática Igual representada por meio de razões, logaritmos, cents e frequências.

\begin{tabular}{|l|c|c|c|c|c|c|c|c|c|c|c|c|c|}
\hline Escala Igual & Lá 3 & Sib3 & Si3 & Dó4 & Dó\# 4 & Ré 4 & Ré\# 4 & Mi 4 & Fá4 & Fá\# 4 & Sol 4 & Sol\# 4 & Lá 4 \\
\hline Razão & & $2^{\frac{1}{12}}$ & $2^{\frac{2}{12}}$ & $2^{\frac{3}{12}}$ & $2^{\frac{4}{12}}$ & $2^{\frac{5}{12}}$ & $2^{\frac{6}{12}}$ & $2^{\frac{7}{12}}$ & $2^{\frac{8}{12}}$ & $2^{\frac{9}{12}}$ & $2^{\frac{10}{12}}$ & $2^{\frac{10}{12}}$ & $2^{\frac{12}{12}}$ \\
\hline Logar. & 1,0000 & 1,0595 & 1,0595 & 1,0595 & 1,0595 & 1,0595 & 1,0595 & 1,0595 & 1,0595 & 1,0595 & 1,0595 & 1,0595 & 1,0595 \\
\hline Cents & 0 & 100 & 200 & 300 & 400 & 500 & 600 & 700 & 800 & 900 & 1000 & 1100 & 1200 \\
\hline Freq. & 442,00 & 468,28 & 496,13 & 525,63 & 556,88 & 590,00 & 625,08 & 662,25 & 701,63 & 743,35 & 787,55 & 834,38 & 884,00 \\
\hline
\end{tabular}

\section{Frequência de referência em Hertz $(F R H z)$}

Na área de performance musical, o nome de Hertz encontra-se fortemente associado à escolha de uma nota de referência para a afinação de um instrumento ou de um conjunto musical. A referência A $=440 \mathrm{~Hz}$ indica que a nota Lá3, com frequência de 440 ciclos por segundo, será utilizada como parâmetro para a afinação de um instrumento. Vários países escolhem parâmetros de afinação que refletem valores culturais. Nos EUA, utiliza-se o A = 440, enquanto na Alemanha pode-se utilizar $\mathrm{A}=443$ ou $\mathrm{A}=444$. No Brasil, devido ao nosso clima predominantemente tropical, opta-se por utilizar $\mathrm{A}=442$ na maioria das orquestras sinfônicas e escolas de música.

Heinrich Hertz (1857-1894) foi aluno de Hermann von Helmholtz no Instituto de Física de Berlim desde 1878. Suas pesquisas ofereceram provas conclusivas sobre a existência de ondas magnéticas (HOFFMAN, 1998). Os estudos de Hertz foram muito importantes para as áreas de eletromagnetismo, transmissão de ondas e radiação. A Conferénce générale des poids e mesures - CGPM, ${ }^{3}$ de 1960, fez uma alteração no termo de indicação de frequências, substituindo ciclos por segundo (cps) pelo termo Hertz (Hz), em homenagem ao físico alemão.

Atualmente, os músicos têm amplo acesso a uma série de afinadores eletrônicos e aplicativos de afinação para tablets e telefones que têm sido incorporados às práticas musicais de profissionais, de estudantes e de amadores. A prática instrumental com o auxílio de afinador, programa de computador ou aplicativo digital tornou-se um hábito na contemporaneidade, e muitos músicos optam por essas ferramentas de afinação pela sua praticidade e eficiência. O padrão de análise das frequências das notas é a nota $\mathrm{A}=440 \mathrm{~Hz}$ aplicado ao Sistema Igual, sendo que alguns aplicativos oferecem a possibilidade de escolha do sistema Pitagórico, Justo ou Mesotônico. Uma das principais escolhas na utilização de afinadores eletrônicos é a determinação de uma frequência de referência de afinação em Hertz definida, neste trabalho, como $\mathrm{FRHz} .{ }^{4}$ A partir de uma $\mathrm{FRHz}$, o músico pode praticar a performance com afinação padrão brasileira em $\mathrm{A}=442 \mathrm{~Hz}$, com afinação mais baixa em $\mathrm{A}$ $=440 \mathrm{~Hz}$, ou com afinação mais alta em A $=444 \mathrm{~Hz}$. A observação da prática adotada por músicos profissionais e por estudantes mostra que a utilização de afinadores eletrônicos pressupõe que o sistema adotado pelo aparelho reflete um padrão de afinação aceito internacionalmente. Desta maneira, os afinadores eletrônicos e, consequentemente, as $\mathrm{FRHz}$ são as principais referências a partir das quais os músicos podem construir os ajustes da afinação no seu próprio instrumento. 
Quando uma indicação mostra que uma nota precisa ser afinada tendo como referência $A=438$, o músico pode experienciar, com o auxílio de um afinador, que esta nota deve ser tocada com afinação mais baixa que o padrão de costume. Já em outra situação, com indicação $\mathrm{A}=445$, sabe-se que esta nota deverá ser afinada muito acima do padrão. Desta maneira, a $F R H z$ serve como parâmetro que utiliza a experiência e a memória de afinações anteriores realizadas com auxílio de afinadores eletrônicos, para indicar os ajustes necessários para a afinação no Sistema Pitagórico ou no Sistema Justo. A partir de um parâmetro fixo e bem estabelecido no Sistema Igual, torna-se possível desenvolver a habilidade de realizar a afinação nos outros sistemas.

\section{Resultados}

Cada método de descrição de sistema de afinação apresenta aspectos específicos para identificação das frequências das notas. Cada sistema apresenta princípios para a definição da sonoridade dos intervalos, e a comparação entre os diversos sistemas e métodos oferece a possibilidade para o músico compreender os procedimentos necessários para efetuar o ajuste na afinação das notas de acordo com os princípios estéticos de cada sistema de afinação. A contribuição específica deste trabalho é a proposta de $\mathrm{FRHz}$ como parâmetro de afinação e elemento de análise para o músico prático. A organização de uma tabela comparativa entre razões e cents foi baseada na proposta de Gunther (2012, p. 376). O processo de associação da frequência de uma nota com um valor de $F R H z$ pode ser realizado a partir da identificação das notas apresentadas na tabela que mostra os valores das razões, frequências, cents e FRHz. (Tabela 6)

Tabela 6: Tabela com a escala baseada na nota Lá3, com a indicação de frequências, razões, cents e $F R H z$.

\begin{tabular}{|c|c|c|c|c|c|c|c|c|c|c|c|c|c|}
\hline \multicolumn{14}{|c|}{ Escala Igual } \\
\hline & Lá 3 & Sib3 & $\mathrm{Si} 3$ & Dó4 & Dó\# 4 & Ré 4 & Ré\# 4 & Mi 4 & Fá4 & Fá\# 4 & Sol 4 & Sol\# 4 & Lá 4 \\
\hline & $1 \mathrm{~J}$ & $2 m$ & $2 M$ & $3 m$ & $3 \mathrm{M}$ & $4 \mathrm{~J}$ & 4aum & $5 \mathrm{~J}$ & $6 m$ & $6 \mathrm{M}$ & $7 m$ & $7 \mathrm{M}$ & $8 \mathrm{~J}$ \\
\hline Razão & $2^{\frac{0}{12}}$ & $2^{\frac{1}{12}}$ & $2^{\frac{2}{12}}$ & $2^{\frac{3}{12}}$ & $2^{\frac{4}{12}}$ & $2^{\frac{5}{12}}$ & $2^{\frac{6}{12}}$ & $2^{\frac{7}{12}}$ & $2^{\frac{8}{12}}$ & $2^{\frac{9}{12}}$ & $2^{\frac{10}{12}}$ & $2^{\frac{10}{12}}$ & $2^{\frac{12}{12}}$ \\
\hline Freq. & 442 & 468,28 & 496,13 & 525,63 & 556,88 & 590 & 625,08 & 662,25 & 701,63 & \begin{tabular}{|l|}
743,35 \\
\end{tabular} & 787,55 & 834,38 & 884 \\
\hline Cents & 0 & 100 & 200 & 300 & 400 & 500 & 600 & 700 & 800 & 900 & 1000 & 1100 & 1200 \\
\hline $\mathrm{FRHz}$ & 442 & 442 & 442 & 442 & 442 & 442 & 442 & 442 & 442 & 442 & 442 & 442 & 442 \\
\hline \multicolumn{14}{|c|}{ Escala Pitagórica } \\
\hline & $1 \mathrm{~J}$ & $2 m$ & $2 \mathrm{M}$ & $3 m$ & $3 \mathrm{M}$ & $4 \mathrm{~J}$ & 4aum & $5 \mathrm{~J}$ & $6 m$ & $6 \mathrm{M}$ & $7 m$ & $7 \mathrm{M}$ & $8 \mathrm{~J}$ \\
\hline Razão & 1 & $56: 243$ & $9: 8$ & $32: 27$ & $81: 64$ & $4: 3$ & 729:512 & $3: 2$ & 128:81 & $27: 16$ & $16: 9$ & $243: 128$ & 2 \\
\hline Freq. & 442,00 & 465,65 & 497,25 & 523,85 & 559,41 & 589,33 & 629,33 & 663 & 698,47 & 745,88 & 785,78 & 839,11 & 884 \\
\hline Cents & 0 & 90 & 204 & 294 & 408 & 498 & 612 & 702 & 792 & 906 & 996 & 1110 & 1200 \\
\hline $\mathrm{FRHz}$ & 442 & 439,5 & 443 & 440,5 & 444 & 441,5 & 445 & 442,5 & 440 & 443,5 & 441 & 444,5 & 442 \\
\hline \multicolumn{14}{|c|}{ Escala Justa } \\
\hline & $1 \mathrm{~J}$ & $2 \mathrm{~m}$ & $2 \mathrm{M}$ & $3 m$ & $3 \mathrm{M}$ & $4 \mathrm{~J}$ & 4aum & $5 \mathrm{~J}$ & $6 m$ & $6 \mathrm{M}$ & $7 m$ & $7 \mathrm{M}$ & $8 \mathrm{~J}$ \\
\hline Razão & 1 & $16: 15$ & $9: 8$ & $6: 5$ & $5: 4$ & $4: 3$ & $45: 32$ & $3: 2$ & $8: 5$ & $5: 3$ & $9: 5$ & $15: 8$ & 2 \\
\hline Freq. & 442 & 471,47 & 497,25 & 530,40 & 552,50 & 586,33 & 621,56 & 663 & 707,20 & 736,67 & 795,60 & 828,75 & 884 \\
\hline Cents & 0 & 112 & 204 & 316 & 386 & 498 & 590 & 702 & 814 & 884 & 1018 & 1088 & 1200 \\
\hline $\mathrm{FRHz}$ & 442 & 445 & $443 \mathrm{~Hz}$ & 446 & 438,5 & 441,5 & 439,5 & 442,5 & 445,5 & 438 & 446,5 & 439 & 442 \\
\hline
\end{tabular}

A análise dos quadros com os valores em termos de razões, frequências, cents e FRHz possibilita a visualização das relações nas proporções entre os valores nos diversos sistemas de afinação. O uso das frequências permite que o músico saiba o número de vibrações exatas para a afinação de uma determinada nota e de acordo com cada um dos sistemas. As razões ilustram a relação que existe entre uma frequência dentro do contexto da 
escala com doze semitons e, além disso, apresentam a relação o centro tonal e cada um dos graus da escala. A representação em cents apresenta como referência um modelo ideal de afinação baseado no Sistema Igual, em que cada nota apresenta um múltiplo exato de cem para cada semitom dentro do contexto da oitava. Dessa maneira, qualquer nota cujo valor difira de um múltiplo de cem necessita ser ajustada de maneira proporcional, ou seja, as notas com valores superiores devem ser ajustadas mais altas, enquanto que as notas com valores inferiores devem ser ajustadas mais baixas. A frequência de referência em $\mathrm{FRHz}$ apresenta um parâmetro comum para a prática musical de instrumentistas e cantores, pois mostra as alterações que existem nos sistemas de afinação e indica quais as modificações devem ser feitas com o intuito de adaptar a afinação de acordo com o sistema desejado.

Ao analisar a escala Pitagórica, torna-se possível observar que a descrição em $\mathrm{FRHz}$ apresenta um valor específico para cada uma das notas (Tabela 7). O processo de afinação tendo como parâmetro a razão 3:2 apresenta o acréscimo de 0,5 Hz para cada novo intervalo de quinta ascendente com as notas: Mi4 = 442,5 (FRHz), Si3 = 443 (FRHz), Fá\#4 = 443,5 $(F R H z)$, Dó\#4 = $444(F R H z)$, Sol\#4 = 444,5 (FRHz) e Ré4 = $445(F R H z)$. Quando se observa um ciclo de quintas descendentes, é possível identificar um decréscimo de 0,5 para cada novo intervalo com as notas: Ré4 $=441,5(F R H z)$, Sol4 $=441(F R H z)$, Dó4 = 440,5 (FRHz), Fá4 $=440(F R H z)$ e Sib3 $=439,5(F R H z)$.

Tabela 7: Escala Pitagórica representada por razões, frequências, cents e $F R H z$.

\begin{tabular}{|c|c|c|c|c|c|c|c|c|c|c|c|c|c|}
\hline \multicolumn{14}{|c|}{ Escala Pitagórica } \\
\hline & Lá 3 & Sib3 & $\mathrm{Si3}$ & Dó4 & Dó\# 4 & Ré 4 & Ré\# 4 & Mi 4 & Fá4 & Fá\# 4 & Sol 4 & Sol\# 4 & Lá 4 \\
\hline & $1 \mathrm{~J}$ & $2 m$ & $2 M$ & $3 m$ & $3 \mathrm{M}$ & $4 \mathrm{~J}$ & 4aum & $5 \mathrm{~J}$ & $6 m$ & $6 \mathrm{M}$ & $7 m$ & $7 \mathrm{M}$ & $8 \mathrm{~J}$ \\
\hline Razão & 1 & $56: 243$ & $9: 8$ & $32: 27$ & $81: 64$ & $4: 3$ & $729: 512$ & $3: 2$ & $128: 81$ & $27: 16$ & $16: 9$ & $243: 128$ & 2 \\
\hline Freq. & 442,00 & 465,65 & 497,25 & 523,85 & 559,41 & 589,33 & 629,33 & 663 & 698,47 & 745,88 & 785,78 & 839,11 & 884 \\
\hline Cents & 0 & 90 & 204 & 294 & 408 & 498 & 612 & 702 & 792 & 906 & 996 & 1110 & 1200 \\
\hline $\mathrm{FRHz}$ & 442 & 439,5 & 443 & 440,5 & 444 & 441,5 & 445 & 442,5 & 440 & 443,5 & 441 & 444,5 & 442 \\
\hline
\end{tabular}

A análise da Escala Justa descrita de acordo com a FRHz também apresenta um cenário no qual todas as notas apresentam valores diferentes em $F R H z$ para cada grau da escala (Tabela 8). Nesta situação, as razões 4:3 ( $4^{\mathrm{a}}$ Justa), 15:8 ( $7^{\mathrm{a}}$ Maior), 5:4 ( $3^{\mathrm{a}}$ Maior) e 5:3 (6 ${ }^{\mathrm{a}}$ Maior) apresentam valores de $\mathrm{FRHz}$ menores do que no Sistema Igual, e devem ser afinadas gradativamente mais baixas. No entanto, as razões 3:2 ( ${ }^{\mathrm{a}}$ Justa), 9:8 (2 $2^{\mathrm{a}}$ Maior), 45:32 (4 $4^{\mathrm{a}} \mathrm{Au}-$ mentada), 16:15 ( $2^{\mathrm{a}}$ menor), 8:5 (6 ${ }^{\mathrm{a}}$ menor), 6:5 ( $3^{\mathrm{a}}$ menor) e 9:5 ( $7^{\mathrm{a}}$ menor) apresentam valores maiores do que o Sistema Igual e devem ser afinadas gradativamente mais altas.

Tabela 8: Escala Justa representada por razões, frequências, cents e $F R H z$.

\begin{tabular}{|c|c|c|c|c|c|c|c|c|c|c|c|c|c|}
\hline \multicolumn{14}{|c|}{ Escala Justa } \\
\hline & Lá 3 & Sib3 & $\mathrm{Si3}$ & Dó4 & Dó\# 4 & Ré 4 & Ré\# 4 & Mi 4 & Fá4 & Fá\# 4 & Sol 4 & Sol\# 4 & Lá 4 \\
\hline & $1 \mathrm{~J}$ & $2 m$ & $2 \mathrm{M}$ & $3 m$ & $3 \mathrm{M}$ & $4 \mathrm{~J}$ & 4aum & $5 \mathrm{~J}$ & $6 m$ & $6 \mathrm{M}$ & $7 m$ & $7 \mathrm{M}$ & $8 \mathrm{~J}$ \\
\hline Razão & 1 & $16: 15$ & $9: 8$ & $6: 5$ & $5: 4$ & $4: 3$ & $45: 32$ & $3: 2$ & $8: 5$ & $5: 3$ & $9: 5$ & $15: 8$ & 2 \\
\hline Freq. & 442 & 471,47 & 497,25 & 530,40 & 552,50 & 586,33 & 621,56 & 663 & 707,20 & 736,67 & 795,60 & 828,75 & 884 \\
\hline Cents & 0 & 112 & 204 & 316 & 386 & 498 & 590 & 702 & 814 & 884 & 1018 & 1088 & 1200 \\
\hline $\mathrm{FRHz}$ & 442 & 445 & $443 \mathrm{~Hz}$ & 446 & 438,5 & 441,5 & 444,5 & 442,5 & 445,5 & 438 & 446,5 & 439 & 442 \\
\hline
\end{tabular}

O contraste entre dois sistemas por meio da comparação entre as notas das escalas Igual e Pitagórica oferece elementos para que seja possível identificar o tipo de ajuste que deverá ser efetuado em cada uma das notas. Quando a escala Igual é comparada com a escala Pitagórica, observa-se que os graus $5 \mathrm{~J}(+0,5)$ e $4 \mathrm{~J}(-0,5)$ apresentam uma proporcionalidade na diferença de 0,5 FRHz. Outros intervalos também apresentam proporcionalidades complementares: $2 \mathrm{M}(+1)$ e $7 \mathrm{~m}(-1), 6 \mathrm{M}(+1,5)$ e $3 \mathrm{~m}(-1,5), 3 \mathrm{M}(+2)$ e $6 \mathrm{~m}(-2), 7 \mathrm{M}(+2,5)$ 
e $2 \mathrm{~m}(-2,5)$, enquanto que a $4^{\mathrm{a}}$ Aumentada apresenta uma diferença de $+3 \mathrm{FRHz}$ (Tabela 7). A interpretação musical por meio da escala pitagórica exige que os graus $2 \mathrm{M}, 6 \mathrm{M}, 3 \mathrm{M} \mathrm{e}$ $7 \mathrm{M}$ sejam afinados sempre mais altos, enquanto que os graus $7 \mathrm{~m}, 3 \mathrm{~m}, 6 \mathrm{~m}$ e $2 \mathrm{~m}$ sejam afinados mais baixos (Tabela 9).

Tabela 9: Diferenças entre escala no Sistema Igual comparado com o Sistema Pitagórico, representados em cents e $F R H z$.

\begin{tabular}{|c|c|c|c|c|c|c|c|c|c|c|c|c|c|}
\hline \multicolumn{14}{|c|}{ Escala Igual } \\
\hline & Lá 3 & Sib3 & $\mathrm{Si3}$ & Dó4 & Dó\# 4 & Ré 4 & Ré\# 4 & Mi 4 & Fá4 & Fá\# 4 & Sol 4 & Sol\# 4 & Lá 4 \\
\hline & $1 \mathrm{~J}$ & $2 m$ & $2 \mathrm{M}$ & $3 m$ & $3 \mathrm{M}$ & $4 \mathrm{~J}$ & 4aum & $5 \mathrm{~J}$ & $6 m$ & $6 \mathrm{M}$ & $7 m$ & $7 \mathrm{M}$ & $8 \mathrm{~J}$ \\
\hline Razão & $2^{\frac{0}{12}}$ & $2^{\frac{1}{12}}$ & $2^{\frac{2}{12}}$ & $2^{\frac{3}{12}}$ & $2^{\frac{4}{12}}$ & $2^{\frac{5}{12}}$ & $2^{\frac{6}{12}}$ & $2^{\frac{7}{12}}$ & $2^{\frac{8}{12}}$ & $2^{\frac{9}{12}}$ & $2^{\frac{10}{12}}$ & $2^{\frac{10}{12}}$ & $2^{\frac{12}{12}}$ \\
\hline Freq. & 442 & 468,28 & 496,13 & 525,63 & 556,88 & 590 & 625,08 & 662,25 & 701,63 & 743,35 & 787,55 & 834,38 & 884 \\
\hline Cents & 0 & 100 & 200 & 300 & 400 & 500 & 600 & 700 & 800 & 900 & 1000 & 1100 & 1200 \\
\hline $\mathrm{FRHz}$ & 442 & 442 & 442 & 442 & 442 & 442 & 442 & 442 & 442 & 442 & 442 & 442 & 442 \\
\hline \multicolumn{14}{|c|}{ Escala Pitagórica } \\
\hline & Lá 3 & Sib3 & $\mathrm{Si3}$ & Dó4 & Dó\# 4 & Ré 4 & Ré\# 4 & Mi 4 & Fá4 & Fá\# 4 & Sol 4 & Sol\# 4 & Lá 4 \\
\hline Razão & 1 & $256: 243$ & $9: 8$ & $32: 27$ & $81: 64$ & $4: 3$ & $729: 512$ & $3: 2$ & 128:81 & $27: 16$ & $16: 9$ & $243: 128$ & 2 \\
\hline Freq. & 442,00 & 465,65 & 497,25 & 523,85 & 559,41 & 589,33 & 629,33 & 663 & \begin{tabular}{|l|}
698,47 \\
\end{tabular} & 745,88 & 785,78 & 839,11 & 884 \\
\hline Cents & 0 & 90 & 204 & \begin{tabular}{|l|}
294 \\
\end{tabular} & 408 & 498 & 612 & 702 & \begin{tabular}{|l|}
792 \\
\end{tabular} & 906 & 996 & 1110 & 1200 \\
\hline $\mathrm{FRHz}$ & 442 & 439,5 & 443 & 440,5 & 444 & 441,5 & 445 & 442,5 & 440 & 443,5 & 441 & 444,5 & 442 \\
\hline $\begin{array}{l}\text { Difer. } \\
\text { Cents }\end{array}$ & 0 & -10 & 4 & -6 & 8 & -2 & 12 & 2 & -8 & 6 & -4 & 10 & 0 \\
\hline $\begin{array}{l}\text { Difer. } \\
\text { FRHz }\end{array}$ & 0 & $-2,5$ & 1 & $-1,5$ & 2 & $-0,5$ & 3 & 0,5 & -2 & 1,5 & -1 & 2,5 & 0 \\
\hline
\end{tabular}

O contraste entre a escala Igual e escala Justa demonstra que as diferenças de afinação são muito grandes entre 1 e $4 \mathrm{FRHz}$, o que exige muita flexibilidade para que seja realizado o temperamento das notas (Tabela 10). O contexto do sistema Justo exige que as 3as correspondentes aos acordes maiores I (3M, -3,5), IV (6M, -4) e V (7M, -3) devem ser muito baixas para permitir uma afinação sem batimentos entre os harmônicos superiores da série harmônica. De maneira complementar, as 3as dos acordes menores serão mais altas nos graus $\operatorname{Im}(3 \mathrm{~m},+4), \operatorname{IVm}(6 \mathrm{~m},+3,5)$ e $\operatorname{Vm}(7 \mathrm{~m},+2,5)$.

Tabela 10: Diferenças entre escalas maiores no Sistema Igual comparado com o Sistema Justo, representados em cents e FRHz.

\begin{tabular}{|c|c|c|c|c|c|c|c|c|c|c|c|c|c|}
\hline \multicolumn{14}{|c|}{ Escala Igual } \\
\hline & Lá 3 & Sib3 & $\mathrm{Si3}$ & Dó4 & Dó\# 4 & Ré 4 & Ré\# 4 & Mi 4 & Fá4 & Fá\# 4 & Sol 4 & Sol\# 4 & Lá 4 \\
\hline & $1 \mathrm{~J}$ & $2 m$ & $2 \mathrm{M}$ & $3 m$ & $3 \mathrm{M}$ & $4 \mathrm{~J}$ & 4aum & $5 \mathrm{~J}$ & $6 \mathrm{~m}$ & $6 \mathrm{M}$ & $7 m$ & $7 \mathrm{M}$ & $8 \mathrm{~J}$ \\
\hline Razão & $2^{\frac{0}{12}}$ & $2^{\frac{1}{12}}$ & $2^{\frac{2}{12}}$ & $2^{\frac{3}{12}}$ & $2^{\frac{4}{12}}$ & $2^{\frac{5}{12}}$ & $2^{\frac{6}{12}}$ & $2^{\frac{7}{12}}$ & $2^{\frac{8}{12}}$ & $2^{\frac{9}{12}}$ & $2^{\frac{10}{12}}$ & $2^{\frac{10}{12}}$ & $2^{\frac{12}{12}}$ \\
\hline Freq. & 442 & 468,28 & 496,13 & 525,63 & 556,88 & 590 & 625,08 & 662,25 & 701,63 & 743,35 & 787,55 & 834,38 & 884 \\
\hline Cents & 0 & 100 & 200 & 300 & 400 & 500 & 600 & \begin{tabular}{|l|}
700 \\
\end{tabular} & 800 & 900 & 1000 & 1100 & 1200 \\
\hline $\mathrm{FRHz}$ & 442 & 442 & 442 & 442 & 442 & 442 & 442 & 442 & 442 & 442 & 442 & 442 & 442 \\
\hline \multicolumn{14}{|c|}{ Escala Justa } \\
\hline & $1 \mathrm{~J}$ & $2 \mathrm{~m}$ & $2 \mathrm{M}$ & $3 m$ & $3 \mathrm{M}$ & $4 \mathrm{~J}$ & 4aum & $5 \mathrm{~J}$ & $6 m$ & $6 \mathrm{M}$ & $7 \mathrm{~m}$ & $7 \mathrm{M}$ & $8 \mathrm{~J}$ \\
\hline Razão & 1 & $16: 15$ & $9: 8$ & $6: 5$ & $5: 4$ & $4: 3$ & $45: 32$ & $3: 2$ & $8: 5$ & $5: 3$ & $9: 5$ & $15: 8$ & 2 \\
\hline Freq. & 442 & 471,47 & 497,25 & 530,40 & 552,50 & 586,33 & 621,56 & 663 & 707,20 & 736,67 & 795,60 & 828,75 & 884 \\
\hline Cents & 0 & 112 & 204 & 316 & 386 & 498 & 590 & 702 & 814 & 884 & 1018 & 1088 & 1200 \\
\hline $\mathrm{FRHz}$ & 442 & 445 & $443 \mathrm{~Hz}$ & 446 & 438,5 & 441,5 & 439,5 & 442,5 & 445,5 & 438 & 446,5 & 439 & 442 \\
\hline $\begin{array}{l}\text { Difer. } \\
\text { Cents }\end{array}$ & 0 & 12 & 4 & 16 & -14 & -2 & -10 & 2 & 14 & -16 & 18 & -12 & 0 \\
\hline $\begin{array}{l}\text { Difer. } \\
\text { FRHz }\end{array}$ & 0 & 3 & 1 & 4 & $-3,5$ & $-0,5$ & $-2,5$ & 0,5 & 3,5 & -4 & 2,5 & -3 & 0 \\
\hline
\end{tabular}


A análise das relações entre os valores da escala Pitagórica e os valores da escala Justa demonstra as maiores diferenças entre todos os sistemas investigados (Tabela 11). As notas da 5J, da 4J e da 2M apresentam razões e valores iguais, enquanto 3M, 4Aum, 6M e 7M têm diferença de -5,5 FRHz; as $2 \mathrm{~m}$, 3m, $6 \mathrm{~m}$ e $7 \mathrm{~m}$ apresentam uma diferença de +5,5 FRHz. Esta diferença de 5,5 Hz ou 22 cents corresponde à coma sintônica, gerada pela diferença entre a razão de uma $3^{\text {a }}$ Maior Pitagórica (81:64) e uma $3^{\text {a }}$ Maior Justa (5:4) (Barbour, 2004). Neste caso, foi possível verificar que a coma sintônica está presente na relação entre todos os intervalos que diferenciam a escala Pitagórica da escala Justa..

Tabela 11: Diferenças entre escalas maiores no Sistema Pitagórico comparado com o Sistema Justo, representados em cents e $\mathrm{FRHz}$.

\begin{tabular}{|c|c|c|c|c|c|c|c|c|c|c|c|c|c|}
\hline \multicolumn{14}{|c|}{ Escala Pitagórica } \\
\hline & Lá 3 & Sib3 & $\mathrm{Si3}$ & Dó4 & Dó\# 4 & Ré 4 & Ré\# 4 & Mi 4 & Fá4 & Fá\# 4 & Sol 4 & Sol\# 4 & Lá 4 \\
\hline & $1 \mathrm{~J}$ & $2 m$ & $2 \mathrm{M}$ & $3 m$ & $3 \mathrm{M}$ & $4 \mathrm{~J}$ & 4aum & $5 \mathrm{~J}$ & $6 m$ & $6 \mathrm{M}$ & $7 \mathrm{~m}$ & $7 \mathrm{M}$ & $8 \mathrm{~J}$ \\
\hline Razão & 1 & $256: 243$ & $9: 8$ & $32: 27$ & $81: 64$ & $4: 3$ & $729: 512$ & $3: 2$ & $128: 81$ & $27: 16$ & $16: 9$ & $243: 128$ & 2 \\
\hline Freq. & 442,00 & 465,65 & 497,25 & 523,85 & 559,41 & 589,33 & 629,33 & 663 & 698,47 & 745,88 & 785,78 & 839,11 & 884 \\
\hline Cents & 0 & 90 & 204 & 294 & 408 & 498 & 612 & 702 & 792 & 906 & 996 & 1110 & 1200 \\
\hline $\mathrm{FRHz}$ & 442 & 439,5 & 443 & 440,5 & 444 & 441,5 & 445 & 442,5 & 440 & 443,5 & 441 & 444,5 & 442 \\
\hline \multicolumn{14}{|c|}{ Escala Justa } \\
\hline & $1 \mathrm{~J}$ & $2 m$ & $2 \mathrm{M}$ & $3 m$ & $3 M$ & $4 \mathrm{~J}$ & 4aum & $5 \mathrm{~J}$ & $6 m$ & $6 \mathrm{M}$ & $7 \mathrm{~m}$ & $7 \mathrm{M}$ & $8 \mathrm{~J}$ \\
\hline Razão & 1 & $16: 15$ & $9: 8$ & $6: 5$ & $5: 4$ & $4: 3$ & $45: 32$ & $3: 2$ & $8: 5$ & $5: 3$ & $9: 5$ & $15: 8$ & 2 \\
\hline Freq. & 442 & 471,47 & 497,25 & 530,40 & 552,50 & 586,33 & 621,56 & 663 & 707,20 & 736,67 & \begin{tabular}{|l|}
795,60 \\
\end{tabular} & 828,75 & 884 \\
\hline Cents & 0 & 112 & 204 & 316 & 386 & 498 & 590 & 702 & 814 & 884 & 1018 & 1088 & 1200 \\
\hline $\mathrm{FRHz}$ & 442 & 445 & $443 \mathrm{~Hz}$ & 446 & 438,5 & 441,5 & 439,5 & 442,5 & 445,5 & 438 & 446,5 & 439 & 442 \\
\hline $\begin{array}{l}\text { Difer. } \\
\text { Cents }\end{array}$ & 0 & 22 & 0 & 22 & -22 & 0 & -22 & 0 & 22 & -22 & 22 & -22 & 0 \\
\hline $\begin{array}{l}\text { Difer. } \\
\text { FRHz }\end{array}$ & 0 & 5,5 & 0 & 5,5 & $-5,5$ & 0 & $-5,5$ & 0 & 5,5 & $-5,5$ & 5,5 & $-5,5$ & 0 \\
\hline
\end{tabular}

Os resultados demonstram que existem variações específicas em todos os graus da escala quando estão sendo utilizados os sistemas Pitagórico, Justo e Igual. A partir da análise das tabelas, torna-se possível estabelecer referências para que instrumentistas possam ajustar e aprimorar sua prática musical na performance dos diferentes sistemas de afinação.

\section{Conclusão}

A performance musical necessita de subsídios para relacionar questões teóricas e transformá-las em procedimentos práticos. Neste artigo, não foram abordados os aspectos históricos ou estéticos de cada sistema de afinação. O objetivo principal foi analisar as diferentes formas de abordagem da afinação e propor um parâmetro prático para a avaliação dos diversos graus da escala de acordo com os sistemas Pitagórico, Justo e Igual. Além disso, os sistemas de afinação foram apresentados de acordo com um parâmetro acessível aos músicos contemporâneos, a $F R H z$. A $F R H z$ pode ser utilizada por qualquer músico que tenha um programa de software ou aplicartivo que possa reconhecer as alturas ou que possa produzir notas musicais nas frequências desejadas. A partir de uma frequência sonora de referência em $\mathrm{FRHz}$, torna-se possível treinar a percepção musical e encontrar os pontos de ajuste na afinação de notas específicas.

A observação de uma escala de doze tons de acordo com quatro parâmetros (frequências, razões, cents e $F R H z$ ) permite a análise da proporcionalidade entre as variações nos valores de cada nota. Nas oscilações entre os valores em Cents X FRHz, observou-se 
que, para cada variação de $1 \mathrm{~Hz}$, havia igual variação de 4 cents, tanto no sentido positivo quanto negativo. Pode-se propor que cada variação de um cent corresponda aproximadamente a uma variação de 0,25 na FRHz. Desta maneira, torna-se possível analisar várias propostas de temperamentos realizados por Ellis (1894) e Gunther (2012) e adequá-las aos parâmetros de $\mathrm{FRHz}$ e, assim, transferir as informações em cents para as práticas atuais de performance.

A relação entre as propostas teóricas apresentadas em razões e cents é fundamental para a compreensão da literatura que aborda os diversos temperamentos musicais. As práticas de afinação dos períodos renascentista, barroco, clássico e romântico foram desenvolvidas antes da consolidação do Sistema Igual como referência para a afinação de instrumentos de afinação fixa, no início do séc. XX. A justaposição dos diferentes pontos de vista e as formas de abordagem da afinação da escala oferecem recursos para o desenvolvimento de procedimentos práticos para o ajuste da afinação de instrumentos de corda e sopro, principalmente em situações nas quais não estão presentes instrumentos de afinação fixa.

O paradoxo apresentado no início deste artigo colocava em oposição a objetividade matemática e a subjetividade perceptiva. No entanto, a objetividade do conhecimento pode complementar a subjetividade das sensações ao organizar e estruturar experiências musicais. Nesse contexto, os parâmetros de afinação oferecem critérios para identificação das frequências desejadas e caberá ao músico desenvolver sua sutileza e sua delicadeza perceptiva para poder apresentar um rico vocabulário de possibilidades de afinação no seu instrumento musical.

\section{Notas}

1 A discussão proposta aqui terá como referência os instrumentos de afinação variável de cordas ou sopro, pois estes instrumentos permitem o controle das frequências das notas produzidas.

2 Neste artigo, todos os exemplos estarão relacionados à frequência padrão Lá $3=442 \mathrm{~Hz}$, pois corresponde ao principal padrão de afinação utilizado no Brasil.

3 Conferência Internacional de Pesos e Medidas - esta conferência faz parte do Bureau Internationale de Poids et Mesures- BIPM, que regulamenta os padrões internacionais para medidas e pesos. http://www.bipm.org:en/worldwide-metrology/cgpm/

4 Em inglês, utiliza-se o termo general tuning standard para se referir à utilização da nota Lá $=440$ Hz como nota de referência para a afinação. No entanto, não existe um termo equivalente em português e, desta forma, foi necessária a elaboração do conceito de FRHz - frequência de referência em Hertz.

\section{Referências}

BARBOUR, J. Murray. Tuning and Temperament: A Historical Survey. New York: Dover, 2004.

ELLIS, Alexander J. e Hipkins, Alfred J. Tonometrical Observations on Some Existing Non-Harmonic Musical Scales. Proceedings of the Royal Society of London, v. 37, p. 368-385, 1884.

GUNTHER, Leon. The Physics of Music and Color. London, New York: Springer, 2012.

HELMHOLTZ, Hermann von. On the Sensations of Tone as a Physiological Basis for the Theory of Music, $3^{\mathrm{a}}$ edição inglesa, traduzida por Alexander J. Ellis. London: Longmans, Green, and Co, 1895. 
HOFFMAN, Dieter. Heinrich Hertz and the Berlin School of Phisics. In: Baird, D., HUGHES, R. I.; NORDMANN, Alfred (Editors). Heinrich Hertz: Classical Physicist, Modern Philosopher. Boston: Luwer Academic Publishers, 1998.

STOCK, Jonathan. Alexander J. Ellis and His Place in the History of Ethnomusicology. Ethnomusicology. Urbana - Il, v. 51, n. 2, p. 306-325, 2007.

THOMPSON, William Forde. Intervals and Scales. In: Diana Deutsch (Ed.). The Psychology of Music. London, San Diego: Elsevier-Academic Press, Third Edition, 2013. p. 107-140.

Ricardo Dourado Freire - Professor da Universidade de Brasília - UnB nas áreas de Clarineta e Teoria Musical, desde 1995. Cursou Mestrado (1994) e Doutorado (2000) na Michigan State University e Bacharelado em Clarineta (1992) na UnB. Exerceu, entre 1996 e 2016, a função de presidente da Associação Brasileira de Clarinetistas e participa do corpo editorial da revista The Clarinet, escrevendo, desde 2010, a coluna "News from Latin America". Participa ativamente em eventos internacionais de Clarinetistas tendo se apresentado em Portugal, EUA, Itália, Suécia, Noruega, Bulgária, Rússia, Venezuela, Colômbia, Peru e Brasil. Realiza pesquisas nas áreas de Cognição Musical, Performance Musical e Educação Musical. 